\title{
Long-term outcomes of treat-and-extend ranibizumab with and without navigated laser for diabetic macular oedema: TREX-DME 3-year results
}

\author{
John F. Payne (10, ${ }^{1}$ Charles C. Wykoff, ${ }^{2}$ W. Lloyd Clark, ${ }^{1}$ Beau B. Bruce, ${ }^{3}$ \\ David S. Boyer, ${ }^{4}$ David M. Brown, ${ }^{5}$ TREX-DME Study Group
}

\begin{abstract}
- Additional material is published online only. To view please visit the journal online (http://dx.doi.org/10.1136 bjophthalmol-2020-316176).

${ }^{1}$ Palmetto Retina Center, West Columbia, South Carolina, USA ${ }^{2}$ Blanton Eye Institute, Houston Methodist Hospital and Weill Cornell Medical College, Retina Consultants of Houston, Houston, Texas, USA ${ }^{3}$ Department of Ophthalmology, Emory University, Atlanta, Georgia, USA

${ }^{4}$ Ophthalmology, University of Southern California, Sherman Oaks, California, USA

${ }^{5}$ The Methodist Hospital, Houston, TX, Houston, Texas, USA
\end{abstract}

\section{Correspondence to} Dr John F. Payne, Palmetto Retina, West Columbia, South Carolina, USA; jpayne@palmettoretina.com

Presented at the annual meeting of the American Academy of Ophthalmology on 30 October 2018 in Chicago, IL.

Received 27 February 2020 Revised 31 March 2020 Accepted 2 April 2020 Published Online First 17 April 2020

\section{ABSTRACT}

Background/aims To evaluate the long-term effects of treat-and-extend dosing of ranibizumab with and without navigated focal laser for diabetic macular oedema (DME).

Methods This is a multicentre, randomised clinical trial where 150 eyes were randomised into three cohorts; Monthly $(n=30)$, TReat and EXtend without macular laser photocoagulation (TREX; $n=60$ ), and treat and extend with angiography-Gulded macular LAser photocoagulation (GILA; $n=60$ ). During the first 2 years, eyes either received ranibizumab $0.3 \mathrm{mg}$ every 4 weeks or underwent treat-and-extend ranibizumab with or without angiography-guided laser therapy. In the third year, all eyes were treated as needed with ranibizumab for $>5$ letters vision loss or if the central retinal thickness (CRT) was $>325 \mu \mathrm{m}$, and all eyes were eligible to receive focal laser.

Results 109 eyes (73\%) completed the 3-year endpoint. At week 156, mean best-corrected visual acuity (BCVA) and CRT improved by 6.9, 9.7, 9.5 letters $(p=0.60)$ and $129,138,165 \mu \mathrm{m}(\mathrm{p}=0.39)$, in the Monthly, TREX and GILA cohorts, respectively. These improvements were reached prior to week 104 and no significant changes occurred from week 104 to week 156 (BCVA: $p=0.34 ; C R T: p=0.36$ ). The mean number of injections in the third year was 3.0,3.1, and 2.4 in the Monthly, TREX and GILA cohorts, respectively $(p=0.56)$. 86 eyes (79\%) required at least one ranibizumab injection in the third year.

Conclusion The improvements achieved after 2 years of treat-and-extend ranibizumab for DME were maintained in the third year with a mean of 3 intravitreal injections. Trial registration number FDA IND 119146, NCT01934556.

\section{INTRODUCTION}

Diabetic macular oedema (DME) continues to be a leading cause of blindness in working-age adults. ${ }^{1}$ Consistent blockade of vascular endothelial growth factor (VEGF) has been shown to be effective at reducing oedema and improving vision in subjects with vision loss due to this disease. ${ }^{2-4}$ Several large clinical trials have shown that most patients who are treated with an initial period of intensive antiVEGF medication can maintain their visual and anatomical gains with less than monthly dosing. ${ }^{5-8}$ It should be noted that while treatment burden is often decreased over longer periods of treatment, a majority of patients continue to require some
anti-VEGF treatment in order to maintain their vision. For example, while the mean number of injections in the Open-Label Extension (OLE) study of the RISE/RIDE clinical trials was 4.5 injections, approximately $75 \%$ of subjects in the OLE study still required some re-treatment. ${ }^{8}$ The ENDURANCE extension trial, which used intravitreal aflibercept, similarly showed that $70 \%$ of subjects required re-treatment over the course of the study. ${ }^{5}$ A question remains as to whether more conservative and consistent dosing initially leads to fewer long-term injections in order to maintain initial visual gains.

Treat-and-extend dosing allows the clinician to titrate anti-VEGF dosing to the individual's disease response, potentially leading to a decrease in treatment burden. Several studies have now demonstrated the effectiveness of this treatment approach for DME for up to 2 years. $^{9-12}$ No prospective data have been reported on the efficacy of a treat and extend dosing strategy beyond 2 years. The TREX-DME trial was designed to assess the efficacy of a treat and extend dosing algorithm of ranibizumab $0.3 \mathrm{mg}$ (Lucentis, Genentech, South San Francisco) with and without navigated focal laser therapy to monthly dosing for DME. ${ }^{9}$ In TREX-DME, all subjects were treated with either monthly or treat and extend dosing for 2 years. In the third year of study, all subjects were then transitioned to a pro re nata (PRN) approach. The purpose of this manuscript is to evaluate the longterm outcomes of subjects in the TREX-DME trial and assess the sustained effect of treat and extend dosing with and without navigated focal laser therapy for DME.

\section{METHODS}

TREX-DME (TReat and EXtend Protocol in Patients with Diabetic Macular Edema) is a phase I/II, multicentre, randomised controlled clinical trial. ${ }^{9}$ All study conduct adhered to the tenets of the Declaration of Helsinki and was in compliance with the Health Insurance Portability and Accountability Act.

The TREX-DME study methods through the first 2 years of study were previously reported. ${ }^{10}$ Briefly, eyes were randomised 1:2:2 into one of three cohorts through a computer algorithm: Monthly cohort ( $n=30$ eyes); treat and extend without angiographyguided focal laser (TREX cohort, $n=60$ eyes); treat and extend with angiography-guided focal laser 
(GILA cohort, $\mathrm{n}=60$ eyes). During the first 2 years, eyes either received ranibizumab $0.3 \mathrm{mg}$ every 4 weeks (Monthly cohort) or underwent a treat and extend dosing regimen of ranibizumab $0.3 \mathrm{mg}$ with or without angiography-guided laser therapy based on pre-specified criteria for disease activity.

In the third year, all eyes were examined every 4 weeks and treated with ranibizumab $0.3 \mathrm{mg}$ on a PRN basis if there was $>5$ letters vision loss compared with the vision at the week 104 visit or if the central retinal thickness (CRT) was $>325 \mu \mathrm{m}$. The CRT was defined as the average retinal thickness of the central $1 \mathrm{~mm}$ around the fovea using Heidelberg Spectralis spectraldomain optical coherence tomography (SD-OCT; Heidelberg Engineering, Heidelberg, Germany). In addition, all eyes were eligible to receive focal laser therapy in the third year. All eyes were evaluated at weeks 116, 128, 140 and 152 and were given angiography-guided focal laser therapy if the eye had received $\geq 2$ intravitreal injections over the previous 90 days. The examining physician could defer focal laser treatment if significant macular ischaemia was present or if the laser treatment was considered to be too close to the foveal avascular zone. All laser treatments were performed on the $532 \mathrm{~nm}$ NAVILAS laser system (OD-OS GmbH, Teltow, Germany).

The primary outcome measures were change in mean bestcorrected visual acuity (BCVA) from screening and week 104 to week 156 . Secondary outcome measures included mean change in CRT, total number of intravitreal injections and laser treatments, percentage of patients gaining or losing 2 and 3 lines of vision, and the incidence and severity of ocular and non-ocular adverse events from week 104 to week 156. A description of the statistical computations, including power calculations, have been previously reported. ${ }^{9}$ The intention-to-treat principle was used in all analyses. Statistical comparisons were performed with R V.3.2.4 (R Project for Statistical Computing, www.rproject.org). Analysis of variance was used to compare continuous outcomes and the $\chi^{2}$ or Fisher exact test, as appropriate, was used to compare categorical outcomes between the three arms of the study. As a sensitivity analysis, multiple imputation using chained equations was carried out for missing ETDRS and SD-OCT measurements using a mixed-effects linear model based on treatment visit week (random slope and intercept) with arm as a fixed effect. One hundred imputed datasets were created with 10 iterations each.

\section{RESULTS}

One hundred fifty eyes were enrolled between November 2013 and April 2015 and baseline demographics at screening were well balanced between the three cohorts. ${ }^{9}$ A total of 109 eyes $(73 \%)$ reached the 3 -year end-point visit at week 156 . Figure 1 shows a flow diagram of study eyes through the 3 years of study. Over the 3 years of study, 19 subjects (26 eyes) were lost to follow-up and 4 subjects (5 eyes) withdrew consent. Eight subjects (10 eyes) died prior to reaching the 3-year end-point visit. One of the deaths was from unknown causes, but none of the other deaths was considered to be related to study medication or study procedure.

\section{Visual acuity outcomes}

The visual acuity improvements at week 104 were maintained in the third year of study (figure 2). At week 156, mean BCVA improved by 6.9, 9.7 and 9.5 letters in the Monthly, TREX and GILA cohorts, respectively, and there were no significant differences between the groups $(p=0.60)$. Visual acuity gains were similar and there was no difference between the cohorts when
32 subjects (193 eyes) assessed for eligibility

eyes did not meet inclusion criteria 2 subjects ( 2 eyes) refused participation

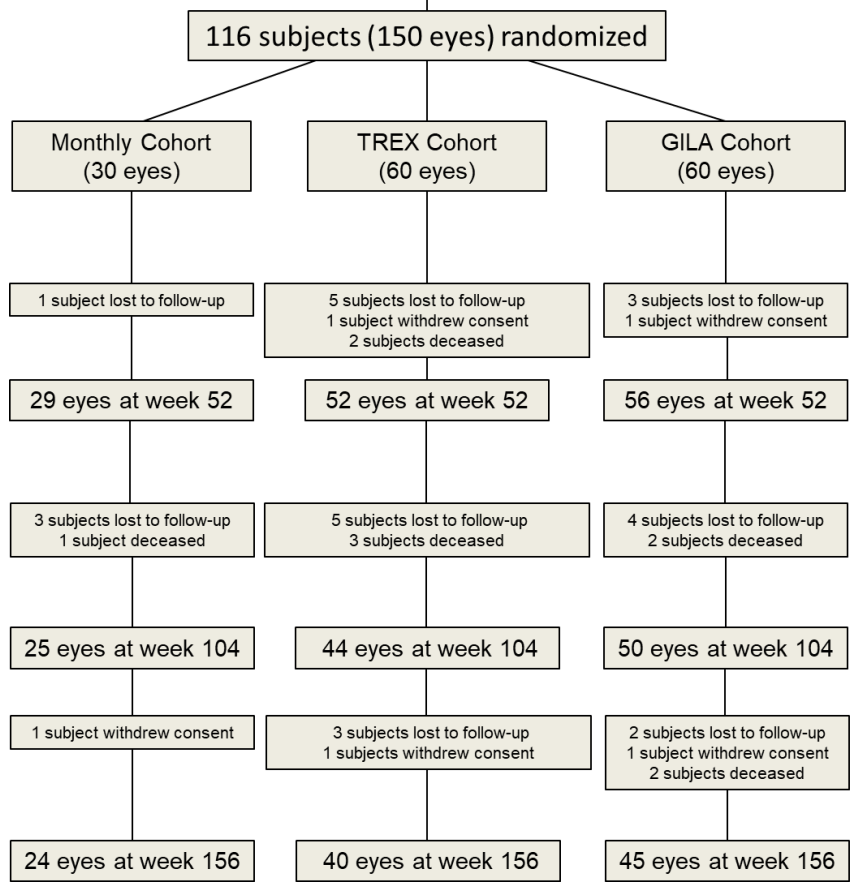

Figure 1 Diagram of patient flow throughout the study from screening to week 156 .

multiple imputations were performed to include eyes which did not reach the 3-year end-point visit.

A similar number of eyes gained 1 line, 2 lines and 3 lines of vision in each of the three cohorts. Of those who reached the 3 -year end-point, 13 (52\%) Monthly eyes, 28 (64\%) TREX eyes and $32(64 \%)$ GILA eyes gained 1 line of vision. Similarly, 12 (48\%) Monthly eyes, 19 (43\%) TREX eyes and 22 (44\%) GILA eyes gained 2 lines of vision. Six (24\%) Monthly eyes, 12 (27\%) TREX eyes and 15 (30\%) GILA eyes gained 3 lines of vision. More eyes in the Monthly cohort lost 1 line and 2 lines of vision compared with the other two cohorts, but it should be noted that these numbers were small. Five (20\%) Monthly eyes lost 1 line of vision compared with 1 (2\%) TREX eye and 3 (6\%) GILA eyes. Two (8\%) Monthly eyes lost 2 lines of vision compared with $1(2 \%)$ TREX eye and no GILA eyes. None of the study eyes lost 3 lines of vision at the 3 -year end-point.

\section{Anatomical outcomes}

The anatomical improvements in CRT prior to week 104 were also maintained at week 156 . At week 156, mean CRT improved by 129,138 and $165 \mu \mathrm{m}$ in the Monthly, TREX and GILA cohorts, respectively, and there were no significant differences between the groups $(p=0.39)$. When multiple imputation was performed to include those eyes not reaching the 3-year endpoint visit, the results were similar. Figure 3 shows a locally weighted regression analysis for change in CRT over time.

\section{Treatment burden}

In the third year, re-treatment with intravitreal ranibizumab was performed if there was $>5$ letters of vision loss compared with the week 104 visit or if the CRT was $>325 \mu \mathrm{m}$. In total, 364 intravitreal injections were given in the third year. Of these, 240 


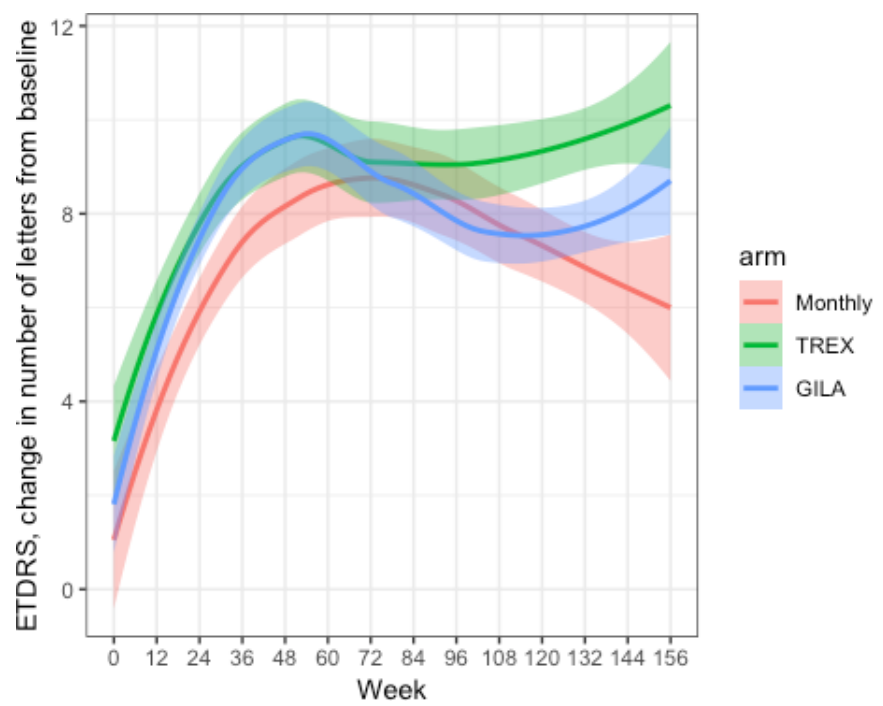

Figure 2 Locally weighted regression analysis for change in visual acuity over time.

injections (66\%) were given because of the anatomical criteria, 57 injections (16\%) were given because of the vision criteria, and 67 injections (18\%) were given because of a combination of both the vision and anatomical criteria. The mean number of intravitreal injections in the third year was 3.0, 3.1 and 2.4 in the Monthly, TREX and GILA cohorts, respectively. There were no significant differences between the groups in the number of injections given in the third year $(p=0.56)$. A majority of eyes still required some treatment with intravitreal injections at some point in the third year. Only 7 (29\%) Monthly eyes, 4 (11\%) TREX eyes and 12 (27\%) GILA eyes received no intravitreal injections during the third year. Conversely, 2 Monthly eyes (8\%), 4 TREX eyes (10\%) and 1 GILA eye (2\%) required intravitreal injections every 4 weeks in the third year due to persistent disease activity. Controlling for arm, baseline and year 2 visual acuity, and baseline CRT, thicker CRT at year 2 predicted a larger number of injections during year 3 (1.2 injections/50 $\mu \mathrm{m}$ increase; $\mathrm{p}<0.0001)$. There were similar visual acuity outcomes

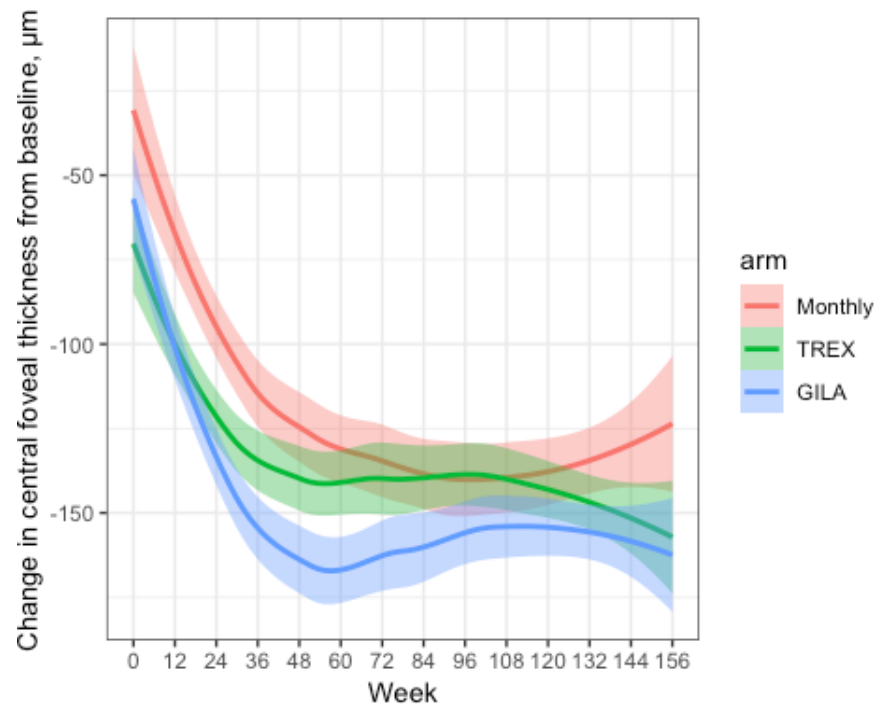

Figure 3 Locally weighted regression analysis for change in central foveal thickness over time. among those who received no injections versus those who received at least one injection.

A total of 37 navigated focal laser treatments were performed in the third year of study. All eyes who met criteria for focal laser therapy in the third year received this treatment. Overall, eyes which required focal laser treatment in the third year had thicker CRT (30 $\mu \mathrm{m}$ on average) at week 104 compared with those eyes which did not require focal laser treatment. This difference was not statistically significant. A similar percentage of TREX eyes (14 eyes, 36\%) required focal laser therapy compared with the Monthly (2 eyes, $8 \%)$ and GILA ( 8 eyes, $18 \%)$ cohorts $(p=0.12)$. A subanalysis was performed on these eyes, and there were no baseline differences between the cohorts for the eyes which required laser during the third year. Among those receiving focal laser therapy, the median number of treatments was 1.5 (range, 1-3) in the TREX, 1.5 (range, 1-3) in the Monthly and 1.0 (range, 1-3) in the GILA cohorts. There were no significant differences between the cohorts in regards to BCVA, CRT or number of injections at week 156 among those who received laser during the third year.

\section{Safety data}

No new safety signals were identified throughout the course of the study. The most common ocular adverse events were blurred vision and posterior vitreous detachment/floaters. Most clinically relevant during the third year, 7 eyes (1 Monthly eye, 4 TREX eyes and 2 GILA eyes) developed new-onset vitreous haemorrhage due to worsening diabetic retinopathy. A complete summary of the adverse events and serious adverse events over the third year of the study are shown in online supplementary tables 1 and 2, respectively.

\section{DISCUSSION}

The TREX-DME study examined a treat and extend dosing algorithm of ranibizumab $0.3 \mathrm{mg}$ for 2 years and then treated patients in a PRN fashion in the third year of study. The current analysis was undertaken to help determine the sustainability of a treat and extend approach for DME. The visual and anatomical improvements achieved after 2 years of treat and extend ranibizumab were maintained in the third year of study with a mean of approximately three intravitreal injections. Furthermore, there was no significant difference in visual or anatomical outcomes, or the number of injections in the third year, when compared with the cohort treated with monthly ranibizumab for 2 years.

Substantial clinical trial data exist demonstrating the beneficial effects of early and consistent treatment with anti-VEGF medications for DME. The DRCR's Protocol I also showed that vision improvement typically occurs, although less robustly, even when the initiation of treatment is delayed. ${ }^{7}$ In that study, eyes that were initially treated with either focal laser therapy or triamcinolone experienced a substantial reduction of central subfield thickness after their first treatment with ranibizumab. ${ }^{7}$ And, while these eyes experienced a 3-4 letter gain after initiating ranibizumab treatment, there was less improvement than was seen in the groups initially receiving ranibizumab at the outset of the study. The 3-year results of the RIDE/RISE clinical trials also showed that a delay in treatment did not result in the same extent of visual improvement as those who were initially treated with monthly ranibizumab. ${ }^{2}$ While TREX-DME did not assess the effect of an initial deferment of anti-VEGF treatment, it did show that an initial treatment with an individualised treat and extend dosing strategy had similar long-term vision improvement and sustainability as those receiving monthly dosing. 
TREX-DME showed a similar treatment burden in the final year of study as those in several other long-term follow-up studies. Vision gains in the final year of TREX-DME were maintained with approximately three intravitreal injections of ranibizumab. Similarly, subjects in the RIDE/RISE OLE study had preserved vision improvements with a mean of 4.5 injections of ranibizumab $0.5 \mathrm{mg}$ over a mean follow-up of 14 months. ${ }^{8}$ The 24-month ENDURANCE extension following the phase III VISTA DME study also demonstrated maintenance of visual gains with a mean of 7.7 intravitreal aflibercept injections through the fourth to fifth years of management. ${ }^{5} 13$ Both of these studies used PRN treatment after the core phase III clinical trial was completed.

An important finding in the long-term results from TREX-DME is that a majority of patients required some level of treatment in order to maintain visual gains. Overall, 79\% of TREX-DME eyes required at least one injection of ranibizumab in the final year of study. This is comparable with the $75 \%$ of eyes in the OLE study and 70\% of eyes in the ENDURANCE study requiring treatment. ${ }^{5}$ The 5 -year Protocol T extension study also found that $70 \%$ of patients required at least one injection between years 2 and $5 .{ }^{14}$ The reduction in anti-VEGF treatment frequency during the third year of TREX-DME associated with transition to PRN re-treatment may have led to under treatment in some cases; seven eyes (6\%) experienced a vitreous haemorrhage due to progression to proliferative diabetic retinopathy. Analogously, worsening of diabetic retinopathy severity has been reported in both the OLE and ENDURANCE studies following transition from fixed dosing to less-frequent PRN antiVEGF re-treatment. ${ }^{5} 8$ These data highlight the importance of strict vigilance and monitoring so that treatment can be rendered as necessary, considering both DME and diabetic retinopathy as indications for intervention.

The 2-year results of TREX-DME demonstrated no significant visual benefits to the addition of navigated focal laser therapy to the treat and extend dosing regimen. ${ }^{10}$ In the third year of this study, all eyes were eligible for focal laser treatment. Overall, eyes that were treated with focal laser therapy had thicker CRT at week 104 compared with those that did not require focal laser treatment. However, the addition of navigated focal laser treatment in these eyes with persistent oedema did not yield significant improvements in vision or anatomy. There was a higher percentage of eyes in the TREX cohort requiring focal laser therapy, but this was not statistically significant $(p=0.12)$. There were also no baseline differences among these eyes and no significant difference in outcomes in these eyes when a subanalysis was performed. It is possible that the deferment of laser treatment for 2 years muted the laser's effect.

A challenge of long-term studies for patients with DME can be retaining patients who have significant systemic comorbidities. The overall retention rate of TREX-DME at 3 years was $73 \%$, which is similar to other large clinical trials. The 3 -year retention rate for the RISE/RIDE clinical trials was between 67\%-80\% and approximately $75 \%$ for the VISTA/VIVID trials. ${ }^{2}{ }^{15}$ Similarly, the 5-year retention rate for the DRCR Network's Protocol I was $77 \%$ and was $67 \%$ for Protocol T extension study. ${ }^{714}$

In conclusion, 2 years of treat and extend ranibizumab dosing using the TREX-DME algorithm resulted in significant vision improvements, which were sustained in the third year of the study with approximately three injections. The addition of navigated focal laser therapy at the initiation of ranibizumab treatment or as a rescue therapy for persistent oedema did not yield significant visual benefits. A majority of patients in TREX-DME and other trials required some longterm treatments with anti-VEGF therapy in order to maintain vision. Long-term physician vigilance is needed for optimal visual outcomes.

Collaborators The following is a list of the members of the TREX-DME Study Group and each were responsible for study protocol administration: Palmetto Retina Center: John F Payne, MD (Chair); W Lloyd Clark, MD; John A Wells, III, MD; David L Johnson, MD; Retina Consultants of Houston: Charles C Wykoff, MD; David M Brown MD; Matthew Benz, MD; Eric Chen, MD; Richard H Fish, MD; Rosa Y Kim, MD; James C Major, Jr., MD; Ronan E O'Malley, MD; Amy C Schefler, MD; Ankoor R Shah, MD; Tien P Wong, MD; Retina-Vitreous Associates Medical Group: David S Boyer, MD; Roger L Novack, MD; Thomas G Chu, MD; Firas Rahhal, MD; Homayoun Tabandeh, MD; Richard H Roe, MD; Pouya N Dayani, MD; David Liao, MD; Alexander Walsh, MD; Daniel D Esmaili, MD.

Contributors JP: Responsible for study design, protocol writing and administration, adverse event monitoring, acquisition and analysis of statistical data, as well as drafting and revising manuscript. CCW: Responsible for study design, protocol writing and administration, acquisition and analysis of statistical data, as well as drafting and revising manuscript. WLC: Responsible for study design, protocol writing and administration, acquisition and analysis of statistical data, as well as drafting and revising manuscript. BB: Responsible for study design, acquisition and analysis of statistical data, as well as drafting and revising manuscript. DSB: Responsible for study design, protocol writing and administration, acquisition and analysis of statistical data, as well as drafting and revising manuscript. DB: Responsible for study design, protocol writing and administration, acquisition and analysis of statistical data, as well as drafting and revising manuscript.

Funding Research grant from Genentech (ML28724).

Disclaimer The funding organisation had no role in the design or conduct of this research.

Competing interests JP: Received research grant funding for TREX-DME from Genentech. Has also received research grant funding from Genentech, Regeneron and the Diabetic Retinopathy Clinical Research Network. CCW: Received personal fees from Acucela, Aerpio, Alimera, Allegro, Allergan, Apellis, Bayer, Chengdu Kanghong, Clearside Biomedical, DORC, Eyepoint, Genentech/Roche, Iveric Bio, Kodiak, Novartis, ONL Therapeutics, Outlook Therapeutics, Oxurion, PolyPhotonics, Recens Medical, Regeneron, Regenxbio, Santen and Takeda. Received research grant funding from Adverum, Aerie Pharmaceuticals, Aerpio, Allergan, Apellis, Chengdu Kanghong, Clearside Biomedical, Gemini Therapeutics, Genentech/Roche, Graybug Vision, IONIS Pharmaceuticals, Iveric Bio, Kodiak, Neurotech, Novartis, Ophthea, Outlook Therapeutics, Recens Medical, Regeneron, Regenxbio, Samsung, Santen and Xbrane Biopharma. WLC: Received personal fees from Regeneron, Genentech, Diabetic Retinopathy Clinical Research Network, Thrombogenics and Ophthea. BB: Received personal fees from Genentech, Emory University, Bayer, Medimmune and individual litigants. DSB: Received personal fees from Alcon, Allegro, Allergan, Bayer, Chengdu Kanghong and Regeneron. DB: Received personal fees from Novartis, Regeneron, Bayer, Genentech/Roche, Adverum, Kodiak, Senju, Chengdu Kanghong, Boehringer Ingelheim, Allegro, Apellis, Gemini, Regenxbio, Stealth, Santen,

Heidelberg, Optos, Navilas, Ocular Therapeutics, iRenix and Lineage Cell. Received research grant funding from Novartis, Regeneron, Bayer, Genentech/Roche, Adverum, Kodiak, Senju, Chengdu Kanghong, Boehringer Ingelheim, Allegro, Apellis, Gemini, Regenxbio, Stealth, Santen, Heidelberg, Optos, Navilas, NEI, NGM, Ionis, Outlook Therapeutics, Xbrane Biopharma and Graybug Vision.

Patient consent for publication Not required.

Ethics approval A centralised institutional review board (Sterling IRB) approved all study protocols and procedures.

Provenance and peer review Not commissioned; externally peer reviewed. Data availability statement Data are available on reasonable request.

\section{ORCID iD}

John F. Payne http://orcid.org/0000-0002-5403-0278

\section{REFERENCES}

1 Ciulla TA, Amador AG, Zinman B. Diabetic retinopathy and diabetic macular edema: pathophysiology, screening, and novel therapies. Diabetes Care 2003;26:2653-64.

2 Brown DM, Nguyen QD, Marcus DM, et al. Long-term outcomes of ranibizumab therapy for diabetic macular edema: the 36-month results from two phase III trials: RISE and RIDE. Ophthalmology 2013;120:2013-22.

3 Wells JA, Glassman AR, Ayala AR, et al. Aflibercept, bevacizumab, or ranibizumab for diabetic macular edema: two-year results from a comparative effectiveness randomized clinical trial. Ophthalmology 2016;123:1351-9. 
4 Brown DM, Schmidt-Erfurth U, Do DV, et al. Intravitreal aflibercept for diabetic macular edema: 100-week results from the VISTA and VIVID studies. Ophthalmology 2015; 122:2044-52.

5 Wykoff CC, Le RT, Khurana RN, et al. Outcomes with as-needed aflibercept and macular laser following the phase III VISTA DME trial: ENDURANCE 12-month extension study. Am J Ophthalmol 2017;173:56-63.

6 Lang GE, Berta A, Eldem BM, et al. Two-year safety and efficacy of ranibizumab 0.5 $\mathrm{mg}$ in diabetic macular edema: interim analysis of the RESTORE extension study. Ophthalmology 2013;120:2004-12.

7 Elman MJ, Ayala A, Bressler NM, et al. Intravitreal ranibizumab for diabetic macular edema with prompt versus deferred laser treatment: 5 -year randomized trial results. Ophthalmology 2015;122:375-81.

8 Boyer DS, Nguyen QD, Brown DM, et al. Outcomes with as-needed ranibizumab after initial monthly therapy: long-term outcomes of the phase III RIDE and RISE trials. Ophthalmology 2015;122:2504-13.

9 Payne JF, Wykoff CC, Clark WL, et al. Randomized trial of treat and extend ranibizumab with and without navigated laser for diabetic macular edema: TREX-DME 1 year outcomes. Ophthalmology 2017;124:74-81.
10 Payne JF, Wykoff CC, Clark WL, et al. Randomized trial of treat and extend ranibizumab with and without navigated laser versus monthly dosing for diabetic macular edema: TREX-DME 2-year outcomes. Am J Ophthalmol 2019;202:91-9.

11 Prünte C, Fajnkuchen F, Mahmood S, et al. Ranibizumab 0.5 mg treat-andextend regimen for diabetic macular oedema: the RETAIN study. Br J Ophthalmol 2016;100:787-95.

12 Ehlers JP, Wang K, Singh RP, et al. A prospective randomized comparative dosing trial of ranibizumab in bevacizumab-resistant diabetic macular edema: the REACT study. Ophthalmol Retina 2018;2:217-24.

13 Wykoff CC, Ou WC, Khurana RN, et al. Long-term outcomes with as-needed aflibercept in diabetic macular oedema: 2-year outcomes of the endurance extension study. Br J Ophthalmol 2018;102:631-6.

14 Wells JA. 5 year outcomes after aflibercept, bevacizumab, or ranibizumab for DME: protocol T extension study. San Francisco, CA: American Academy of Ophthalmology Annual Meeting, 2019.

15 Heier JS, Korobelnik J-F, Brown DM, et al. Intravitreal aflibercept for diabetic macular edema: 148-week results from the VISTA and VIVID studies. Ophthalmology 2016;123:2376-85. 